\title{
Mobile Agent System Framework Suitable for Scalable Networks
}

Francisco Maciá-Pérez

Juan Manuel García-Chamizo 


\title{
Mobile Agent System Framework Suitable for Scalable Networks
}

Francisco Maciá Pérez received his BSc degree in Computer Science in 1994 and the PhD degree in Computer Science in 2001, both by the University of Alicante (Spain). He worked as System Administrator at this same University until 2001. Currently, he is Professor and Principal at the Computer Science and Technology Department at the University of Alicante. His research interests are in the area of network management, computer networks and architectures, distributed systems, embedded devices, mobile agents and web services; areas in those that he directs a very diligent research group.

\author{
Departamento de Tecnología Informática y Computación \\ University of Alicante \\ Apdo. 99, E-03080 \\ Alicante, Spain \\ pmacia@dtic.ua.es \\ TLF.: +34965903979 \\ FAX: +34965909643
}

Juan M. García Chamizo received his BSc in Physics at the University of Granada (Spain) in 1980, and the PhD degree in Computer Science at the University of Alicante (Spain) in 1994. He is currently a Professor at the Department of "Tecnología Informática y Computación” at the University of Alicante. His current research interests are computer vision, reconfigurable hardware, biomedical applications, computer networks and architectures and artificial neural networks. Dr. García Chamizo has directed several research projects related to the above mentioned interest areas. He is a member of a Spanish Consulting Commission on Electronics, Computer Science and Communications. He is also member and editor of some program committees conferences.

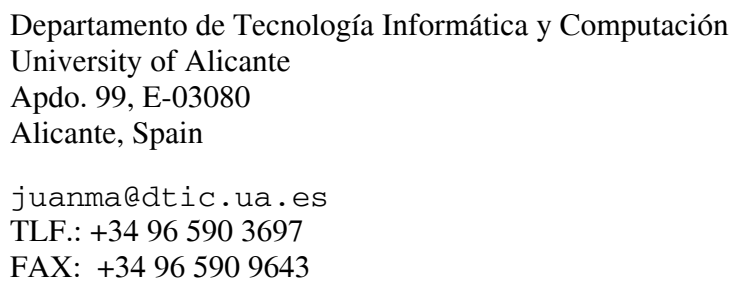




\title{
Mobile Agent System Framework Suitable
}

\section{for Scalable Networks}

\begin{abstract}
In this article we present a formal framework based on the action and reaction model that allows us to cover the dynamics of multi-agent systems (MAS) made up of mobile software agents suitable for scalable networks. This model is based on the operation of the human nervous centres. At the present time, we are applying it in works related with the control of biological systems and also in those related to the network management. In the case of systems based on mobile agents, the main problem is the different vision the agents have of the world and the impossibility of being aware of and synchronizing all the influences brought by the different agents acting on it. We have compared our proposal with the conventional MAS by solving an extension of the predator-prey problem. The results show the advantages of mobility as the size of the problem grows in a distributed system.
\end{abstract}

Keywords: Mobile Agents, Multi-Agent Systems, Communication Networks, Formal Framework. 


\section{Introduction}

In the current communications environment, there is a tendency towards more and more heterogeneous networks. This diversity means that network managers are handling more data and are required to compile huge amounts of information that must be analyzed before undertaking the actual management task. In addition, we also find that present-day network users increasingly expect a reliable and high quality of service. Of course, the ability to provide these guarantees depends on the dynamics of the state of the network, which are closely related to the types of traffic that users generate as part of their communications dialogues. Generally speaking, it requires the implementation of highly sophisticated control and signal techniques (Quendt, 1997), which represents the greatest obstacle to network integration.

These are the main arguments determining research into mobile software agents applied to communications network management (Berners, Hendler and Lassila, 2001). However, although formalisms that provide a suitable formulation for specifying multi-agent systems (MAS) can be found in reading matter, we cannot find formalisms that provide for the peculiarities produced by mobile agents.

Throughout this article, we will describe the background relative to the basic formulation for this type of framework in order to subsequently present our formalism based on the action and reaction model (Ferber, 1999). Once the framework for the performance environment has been established, we will then define the agent model that will inhabit it, and more specifically, focus on the hysteretic agent model proposed by Genesereth and Nilsson (Genesereth and Nilsson, 1987). Taking this model as a basis, we will propose the modifications required for mobile software agents. We 
will subsequently present the model that must define the operation dynamics for a multi-agent system made up of multiple hysteretic software agents. From this formulation, we will propose a set of refinements that will reduce the effect of possible space and time inconsistencies between the agents and the medium, and at the same time, achieve the generalization of the reaction function. We will propose a series of studies and experiments carried out with this model applied to a predator-pray problem variation and finally, we will present the conclusions drawn from this study and future lines of research arising from it.

\section{Background}

Although we can find several formalisms for the representation and reasoning of concurrent processes (Fisher, 1995), (Hoare, 1986), they are of an extremely low level so that, generally speaking, they don't allow agents to be specified in mental state terms, nor do they represent actions explicitly in terms of their effects on the world (Ferber, 1999).

Unlike the classical conception proposed by AI, in which the manifestation of intelligence is based on logical reasoning (Bond and Gasser, 1998), MAS take as their starting point the agents' own behaviour, from the actions they carry out in the world and the interaction among themselves. An action is, above all, a modification.

Anyway, although this is currently one of the most visible theories in the field of AI (Rao and Georgeff, 1991), it proves to be inadequate for situations in which there may be several agents carrying out different activities at the same time and in which they can find themselves in a situation of conflict. 
This problem can be tackled by considering an action as a way of trying to influence the environment, by modifying it according to the agent's goal. However, the consequences of this action needn't be reflected in the world according to its intentions: i.e., the actions carried out by agents must be separated from the effect that they really produce on the states of the world. Especially if we consider that, although actions are not produced explicitly on a specific world, its state is not immutable. In fact, the world is clearly in continuous evolution and keeps on changing without us having to assume the existence of external actions (Ferber, 1999).

There are many ways of modelling actions and their consequences on the world. We will start out from an extension of the action model as a transformation of a global state, based on influences and reactions to influence. This extension provides a new model, known as the action as a response to influences, proposed by (Ferber and Müller, 1995).

In the rest of the article, we will present our formalism constructively, and whenever possible, based on current models.

\section{Formal Framework}

In order to define the complete framework, we have divided its specification into three sections: specification of the agents' environment —-their world—, specification of the agents themselves, and specification of a system consists of a world and multiple agents acting on it. The formulation used is based on the proposal for the action and reaction model (Genesereth and Nilsson, 1987). 


\subsection{Action and Reaction System}

Let us suppose that it is possible to characterize the set $\Sigma=\left\{\sigma_{1}, \sigma_{2}, \ldots\right\}$ of possible world states. Using the algebraic notation specified by Pednault $(1986,1989)$ for ADL (Agent Dynamic Language) language, each world state can be defined by means of the structure $\sigma=\langle D, R, F, C\rangle$. If we bear in mind that the domain and constants remain unaltered for all states, each world state $\sigma_{i}$ could be defined by means of the structure: $\sigma_{i}=\left\langle D, R_{i}, F_{i}, C\right\rangle$

Let us now suppose that we have a finite set $P$ with all the possible tasks that can be carried out in a certain world. We will call each subset $\wp(P) \subset P$ a plan. Since a plan can be made up of one simple task or a set of tasks, from now on we will use the terms plan or task without distinction. In the same way, each plan could be made up of all the tasks $P$, so that both sets would be totally interchangeable in our formulation. However, for reasons of generality, we will use the set $P$. We define the operators who perform the different tasks with a syntax similar to the one for STRIP (Waldinger, 1977) operators, so that each operator will have the form $p=\langle$ name, pre, post $\rangle$, being:

name: This is an expression with the form $f\left(x_{1}, \ldots, x_{k}\right)$, in which each $x_{i}$ are variable authorized to appear in pre and post formulas.

pre: This represents a boolean formula that must be true in order for it to carry out the activity defined in post. 
post: This represents a formula that defines the functionality of the task to be carried out together with the influence it aims to have on the world's new state.

Although this model can be regarded as an extension of the state transformation model, as we shall see, its main difference is that it will enable us to get a separate description of the desired objectives and the real effect produced in the environment. It is precisely this difference that enables us to study the execution of simultaneous actions in the same environment. In order to model this situation, we can define the set $\Gamma=\left\{\gamma_{1}, \gamma_{2}, \ldots\right\}$ for the possible influences or action attempts from the different agents with reference to the current state of the world. This structure is also described as a set of atomic formulas defined with the help of the world states themselves.

In this context, the actions are the result of the combination of the different contributions in the form of influences and the environment's reaction to them. In this way, the execution of a task $p \in P$ modifies the state of the world but we model it as a partially defined application, in which the result is not a new world state but an influence $\gamma \in \Gamma$ on it:

According to this application, a task $p \in P$ can be executed in $\Sigma$, if and only if the application is defined for a specific state $\sigma \in \Sigma$ of the world. We formally express this fact with the predicate:

$$
\gamma=\operatorname{Exec}(p, \sigma)
$$

The function Exec acts in the following way: 


$$
\operatorname{Exec}(\langle\text { name, pre, post }\rangle, \sigma)=\left\{\begin{array}{l}
\text { if pre }(\sigma), \text { post } \\
\text { else }\{\}
\end{array}\right.
$$

Since an influence can be the result of the simultaneity of actions carried out for a specific world state, we can extend the function Exec in order to provide for this fact. To do this, we define the simultaneity operator as $\|$. This operator combines simultaneous actions and gathers the different influences produced by each one of these in a vector. We now carry out the extension of the function by means of a morphism of the action space, equipped with the simultaneity operator $\|$, acting on the set of influence $\Gamma$. Formally:

$$
\operatorname{Exec}:(P, \|) \times \Sigma \rightarrow \Gamma
$$

However, since the aim being sought with task execution is the transition from one world state to another, we must define a world reaction function for the different influences. Thus, the laws of the universe will be described by the application:

$$
\text { React }: \Sigma \times \Gamma \rightarrow \Sigma
$$

This application will be dependent on each type of environment and will have to be defined for each case. Finally, by using the definitions already proposed, we can describe the environment as a system of actions by means of the structure:

$$
\langle\Sigma, \Gamma, P, \text { Exec, React }\rangle
$$

Once the formal framework for the environment based on the action and reaction model has been established, we will present the agents as entities that are capable of influencing the world and distinguishing, from among all its states, the ones that are of interest for the tasks they must perform. 


\subsection{Agents}

We will begin our definitions by focusing on the agent models proposed by Genesereth and Nilsson (1987), pioneers in offering an algebraic representation of their structure and behaviour. However, since this definition was developed on the concept of states, it can only be applied to mono-agent systems. To solve this, we will apply the influence and reaction model (Ferber and Müller, 1995). In spite of this, this model still fails to contemplate the possibility of multiple mobile software agents, so we will introduce the necessary modifications in order to solve this new problem. These modifications basically concentrate on the definition of an execution function disconnected as far as possible from the environment.

Take in Figure 1

As we mentioned in the introduction, we started out from the idea of agents as entities that are permanently perceiving, deliberating and executing; that is, an agent made up of three very different parts: perception, deliberation and execution (Fig. 1). Each agent $\sigma$ is defined by the structure:

$$
\alpha=\langle\text { Domain, Perception, Deliberation }\rangle
$$

In which:

Domain: This refers to the set of elements that make up the structure's domain and represent the perception the agent has of the world in which it is immersed.

Perception: This refers to the set of functions that enable the significant states of the world to be understood and classified. 
Deliberation: This represents the set of functions that make tasks selection possible.

For an agent, perception represents the quality of being able to classify and distinguish different world states; not only with regard to the environment's most significant characteristics, but also with regard to the actions that are its responsibility. We can regard perception as a function that associates a set of values called perceptions or stimuli — perception when speaking of hysteretic agents and stimuli for tropistic agents - with a set of world states $\Sigma$. If we define the set $\Phi_{\alpha}=\left\{\phi_{1}, \phi_{2}, \ldots\right\}$ of possible perceptions associated with agent $\alpha$, the agent's perception function can be defined as:

$$
\operatorname{Percept}_{\alpha}: \Sigma \rightarrow \Phi_{\alpha}
$$

Finally, the capacity for deliberation remains to be defined. Situated between the agent's input and output, it is the element responsible for its current behaviour. It is one of the most complex sections, in which we will define the goals, decision-making and memory faculties, if they have a memory, together with the representation of the world and the concepts used to decide what action to take. According to our definition of this behaviour, we can distinguish two very general types of agent: tropistic and hysteretic agents (Ferber and Müller, 1995). The first one refers to agents that are motivated by stimuli that are not perceived by the conscience; so they will be unable to memorize them. The second type possesses behaviours that will be as sophisticated as we want and that use their previous experience to anticipate the future. For reasons of space and assuming that tropistic agents can be a specific case of hysteretic agents, we will focus our attention on the formulation of the latter. 


\subsubsection{Hysteretic Agents}

An agent of this type is characterized by the fact that it has an internal state that gives it the capacity to memorize and carry out a more valuable decision function than the one studied in the case of tropistic agents.

Take in Figure 2

If we define $S_{\alpha}$ as the set of internal states of a certain agent $\alpha$, a hysteretic agent, within the same action system, (eq. 6), can be described as using the structure:

$$
\alpha=\left\langle\Phi_{\alpha}, S_{\alpha}, \text { Percept }_{\alpha}, \text { Mem }_{\alpha}, \text { Decision }_{\alpha}\right\rangle
$$

That is to say, as a set of perceptions $\Phi_{\alpha}$, a set of internal states $S_{\alpha}$ and a series of functions that model perception, memorization and decision-making on behalf of the agent (Fig. 2). The agent's deliberation capacity is defined in a more complex way by using two different functions: one for memorization and another for decision. The memorization of data consists of going from one internal state to another, so that the memorization function will associate with the agent's internal state and its present perception of the world with a new internal state:

$$
\operatorname{Mem}_{\alpha}: \Phi_{\alpha} \times S_{\alpha} \rightarrow S_{\alpha}
$$

The decision function is responsible for associating a given task to be carried out by the agent from its perception of the world with the internal state in which it is found.

$$
\text { Decision }_{\alpha}: \Phi_{\alpha} \times S_{\alpha} \rightarrow P
$$


In this case, the function that defines the agent's behaviour will have to associate a pair made up of a world state and the agent's internal state, with another pair make up of the action produced together with the agent's new internal state.

$$
\text { Behave }_{\alpha}: \Sigma \times S_{\alpha} \rightarrow P \times S_{\alpha}
$$

For example:

$$
\begin{gathered}
\text { Behave }_{\alpha}(\sigma, s)=\left\langle\operatorname{Decisión}_{\alpha}(\phi, s), \operatorname{Mem}_{\alpha}(\phi, s)\right\rangle \\
\text { with } \phi=\operatorname{Percept}_{\alpha}(\sigma)
\end{gathered}
$$

The result of a given behaviour for a world state and the agent's internal state will be the result of decision and memorization from the perception that this agent has of the state of the world.

\subsubsection{Hysteretic Mobile Agents}

A hysteretic mobile agent is, above all, a hysteretic agent. Therefore, the definitions given in the previous section are perfectly valid for them. However, the notion of mobility implies the possibility that the agents may have to act in conditions with a lack of data. In these cases, the notion of autonomy implicit in the agents and which they must exhibit if we want the system to continue to evolve normally becomes more obvious.

Take in Figure 3

In order to make this task easier, our proposal focuses on including the execution function within the agent's own structure (Fig. 3), so that the structure will have the following form: 


$$
\alpha=\left\langle\Phi_{\alpha}, S_{\alpha}, \text { Percept }_{\alpha}, \text { Mem }_{\alpha}, \text { Decision }_{\alpha}, \text { Exec }_{\alpha}\right\rangle
$$

Thus, we regard the agent as a true PDE agent, maintaining the previously defined components and adding an execution function. This movement would be merely strategic if it didn't separate the agent as far as possible from its environment. To do this, the execution function will operate on the perception the agent has of this environment, instead of operating on a world state. Formally:

$$
\text { Exec }_{\alpha}: P \times \Phi_{\alpha} \rightarrow \Gamma
$$

This new function $\operatorname{Exec}_{\alpha}$ has the same operation mode defined in (eq. 3). The changes introduced in the agent's structure motivate changes in the environment's structure and above all, in the operation dynamics of a multi-agent system. In the following section we will study these implications in more detail.

\subsection{Multiple Mobile Agent System}

Considering the possibility that there is more than one agent inhabiting the world, i.e., a system based on multiple hysteretic mobile software agents, we can represent it by means of the structure:

$$
M M A S=\langle A G, \Sigma, \Gamma, P, \text { React }\rangle
$$

In which $A G=\left\{\alpha_{1}, \alpha_{2}, \ldots\right\}$ represents the system's set of hysteretic software agents and the rest of the elements involved are defined in the same way as in the previous sections.

According to this structure, the system's dynamics are defined by $\operatorname{card}(A G)+1$ equations in which the first equation describes the state of the environment according 
to the time and behaviour of each agent and the remaining equations correspond to modifications in their internal state.

$$
\begin{aligned}
& \sigma(t+1)=\operatorname{React}\left(\sigma(t), \bigcup_{i=1}^{n} \operatorname{Exec}_{i}\left(\operatorname{Decisión}_{i}\left(\phi_{i}(t), s_{i}(t)\right), \phi_{i}(t)\right)\right) \\
& s_{1}(t+1)=\operatorname{Mem}_{1}\left(\phi_{1}(t), s_{1}(t)\right) \\
& s_{n}(t+1)=\operatorname{Mem}_{n}\left(\phi_{n}(t), s_{n}(t)\right) \\
& \text { with } \phi_{i}(t)=\text { Percept }_{i}(\sigma(t))
\end{aligned}
$$

Fig. 4 shows a graphical representation of a hysteretic MMAS made up of three agents acting in it. Now the problem consists of determining the set of internal states for each agent and describing the decision and memorization functions in such a way that the system's behaviour adapts itself to the designer's perspectives and the desired collective phenomena are shown.

\section{Take in Figure 4}

Although the operation dynamics of the mobile software agents are given in this formulation, the inherent problems in their mobility are not solved. In the following section, we present a study of the most typical problems and propose a series of strategies to minimize them.

\section{Model Refinements}

One of the difficulties encountered when putting the formal model described above into practice is that, due to the nature of the problem, we have a specific agent inca- 
pable of contributing its influence or which, because of a malfunction in one of the agents, the influence it contributes isn't the right one or doesn't reach the world.

In order to eliminate, minimize or at least mitigate these problems and systematize the procedure to define the world reaction function (eq. 5), the main one affected by these problems, there are two fundamental points we can touch upon: the set of influences itself or the world reaction function. We will discuss each of these in greater detail below.

\subsection{Influence vector extension}

This approach focuses on the problems caused directly by inconsistencies in the agents' different contributions to the state of the world. Due to intrinsic aspects of the mobile agents themselves (inconsistent data), time aspects (synchronization problems) and space aspects (partial data), the consistency of the world reaction function must be questioned when faced with the impossibility of ensuring a sufficiently limited input set. In order to solve this problem, we propose the introduction of a heuristic capable of deducing the suitable influence vector from a vector with possible faults.

The dynamics of the hysteretic multi-agent system in (eq. 17) could be condensed in the following way - the model doesn't really depend on the type of agent, however, since we have defined the hysteretic agent as a more general example than tropistic agents, for reasons of clarity, we will only use their formulation:

$$
\sigma(t+1)=\operatorname{React}(\sigma(t), \gamma(t))
$$


We define a heuristic $H_{\Gamma}$ capable of deducing a valid influence vector for the world reaction function from an influence vector provided by the system's agents:

$$
H_{\Gamma}: \Sigma \times \Gamma \rightarrow \Gamma
$$

For example:

$$
\gamma^{\prime}=H_{\Gamma}(\sigma, \gamma)
$$

We can replace the original influence vector in (eq. 18) for the heuristic itself and thus manage to absorb or reduce the different problems of inconsistency, incoherence and ambiguity described above.

$$
\sigma(t+1)=\operatorname{React}\left(\sigma(t), H_{\Gamma}(\sigma(t), \gamma(t))\right)
$$

Although, generally speaking, we could use any classifier as a heuristic, due to the potentially large dispersion that exists among the values for the different vectors and their corresponding states, we have opted for the use of a neural network. This type of tool adapts well to the characteristics of this type of problem as it has a high tolerance to faults and a great facility for modelling non-linear functions (Narendra, 1996).

\subsection{Reaction function extension}

The problem here is the specification of the reaction function (eq. 5), responsible for providing the system's present state based on influences from different agents. Now, apart from the problems mentioned in the previous section, we have others that are more closely related to the actual definition of MAS and the reaction function: Basically, they can be summed up in the impossibility of detaining the inference mechanism of the world states from the influence vectors. At worst, we might have failed to 
detect redundancies or very close relationships among the world states that may produce a process of divergence in the system or we might not even have been able to determine the problems.

For this case, the proposal consists of replacing the world reaction function with a heuristic capable of classifying the input influence vectors on a map of optimum states so that it will subsequently be able to assign one of these states to any possible input influence vector.

\section{Experiments}

In this section, we propose a domain extension for the predator-prey problem based on the Tan work (Tan, 1993) in which a collection of animals that we denominate predators, has as objective to capture the biggest number possible of preys. This extension supposes that each node of the network has its own game (ecosystem) and that the predators can communicate to each other, independently of the ecosystem in which they are, as well as to move amongst such in case of being necessary. Now, to the problems of lack of information, originating ones of the high costs derived from the communications amongst ecosystems are added those, along with the originated ones by the transfer of predators.

Next we presented the results of several simulations in which particular situations are solved comparing them with the results obtained by means of the application of our proposal of mobile agents. In general, the obtained results are very encouraging for our model (Fig. 5). The percentage of captures tends to equal itself in both models 
as it increases the predator density. In the referring thing to the number of movements — local and global— both models follow similar guidelines.

Take in Figure 5

Whereas the costs originated by the communication amongst the different ecosystems let practically depend on the number of nodes (Fig. 6a), the local communication increases slightly (Fig. 6b). Considering that the cost caused by the communication amongst nodes is, with difference, the most elevated of the problem, the model derived from our proposal presents characteristics that make it suitable for scalable systems (Lawrence, 2000). Nevertheless, in spite of the promising results, it must be had in mind that we have chosen a problem that adjusts perfectly to our model because the predators are only able to perceive a very limited window, $s[u, v]$, of its surroundings. It should also be kept in mind that this example reflects many of the problems that are outlined in administration of distributed systems.

Take in Figure 6

\section{Conclusions}

In this paper we have presented a formal framework that enables us to cover the operation dynamics of a MAS made up of mobile software agents with the aim of providing an algebraic model that will enable us to define systems with these characteristics in a more systematic and reliable way. 
The proposed model presents characteristics that allow to drastically reducing the traffic of network by the necessity to maintain constantly updated the system state, with which its application in network surroundings favours its scalability.

Another of the advantages offered by this formal framework is unification among the system's specification phases, its design and implementation, making the introduction of a declarative language possible.

We are currently developing auxiliary devices that will act as an agent coprocessor, reducing the additional load that this type of platform generates in the central processor. Our projects for the immediate future include the design of intelligent networking devices that incorporate this platform in a natural way, by incorporating capacity to analyze software agents at circuit level.

\section{References}

Berners-Lee, T., Hendler, J., Lassila, O. (2001), “The Semantic Web”. Scientific American, 284, 5, pp. 34-43.

Bond A., Gasser L. (eds.), (1998), Readings in Distributed Artificial Intelligence. Morgan Kaufman, San Mateo, California.

Ferber, J., Müller, J. (1995), "Influences and Reaction : A model of situated multiagent systems", in proceedings of the 2nd International Conference on Multi-Agent Systems, AAA!Press, Nara, Japan, pp.72-79.

Ferber, J. (1999), Multi-Agent Systems. An Introduction to Distributed Artificial Intelligence. Addison-Wesley, England. 
Fisher, M. (1995), Towards a semantics for Concurrent MetateM. Modal and Temporal Logics. Springer-Verlag, Germany.

Genesereth, M.R., Nilsson N.J. (1987), Logical Foundations of Artificial Intelligence. Morgan Kaufman, England.

Hoare, C. (1986), Communicating Sequential Processes. Prentice-Hall International, Englewood Cliffs.

Lawrence, G. R. (2000), Beyond Moore's Law: Internet Growth Trends. IEEE Computer, Vol. 33, No. 1, pp. 117-119.

Narendra, K.S. (1996), "Neural networks for control: theory and practice", in proceedings IEEE, Vol. 10, pp.1385-1397.

Pednault, E. (1986), "Formulating Multiagent, Dynamic-World Problems in the Classical Planning Framework", in Timberline, M. Georgerff and A. Lansky (eds.), Reasoning about Actions and Plans., Morgan Kaufman, pp.425-448.

Pednault, E. (1989), “ADL: Exploring the middle ground between STRIPS and the situation calculus", in Ronald Brachman, Hector Levesque, and Raymond Reiter (eds.), proceedings of the First Int'1 Conf. on Principles of Knowledge Representation and Reasoning, pp.324-332.

Quendt, B. (1997), “A Signaling Concept for Multimedia-Applications on an open Telecommunication Market", in proceedings of the 2nd Conference on the Practical Application of Intelligent Agents and Multi-Agent Technology (PAAM'97), London.

Rao, A.S. and Georgeff, M.P. (1991), "Modelling rational agents within a BDI-architecture”, in Fikes y Sandewal (eds.), proceedings of Knowledge Representation and Reasoning (KR\&R91), Morgan Kaufmann Publishers, pp.473-484. 
Tan, M. (1993), "Multi-agent reinforcement learning: Independent vs. cooperative agents", in proceedings of the Tenth International Conference on Machine Learning, pp.330-337.

Waldinger, R. (1977), “Achieving Several Goals Simultaneously”, Machine Intelligence, Cap.8, pp.94-136. 


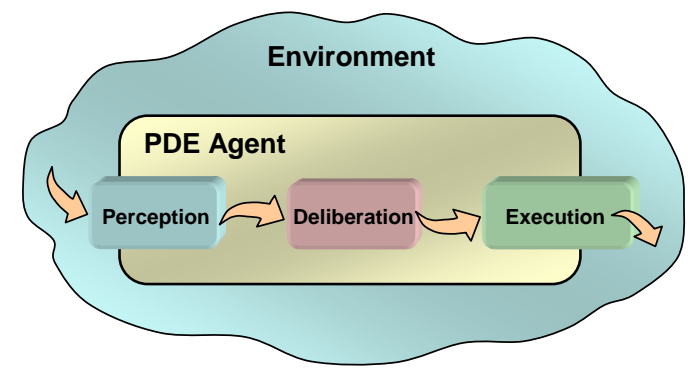

Fig. 1. Agent internal structure: Perception-Deliberation-Execution.

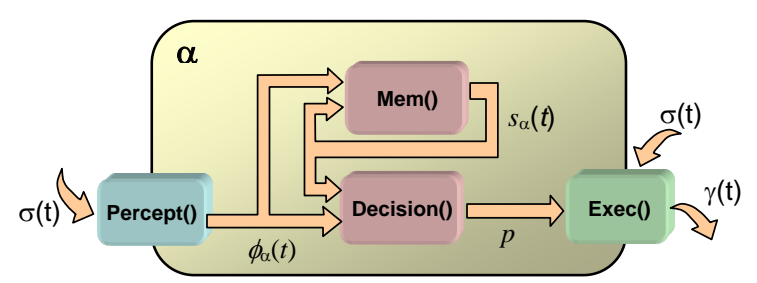

Fig. 2. Graphical representation of a hysteretic agent.

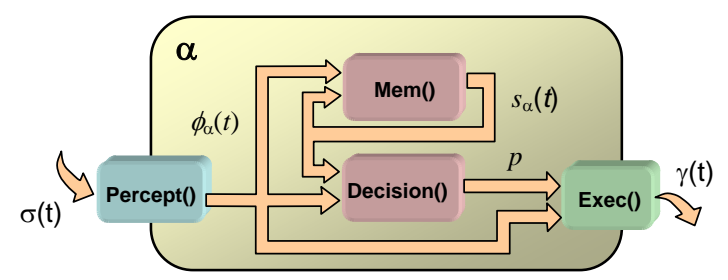

Fig. 3. Graphical representation of a hysteretic mobile agent. 


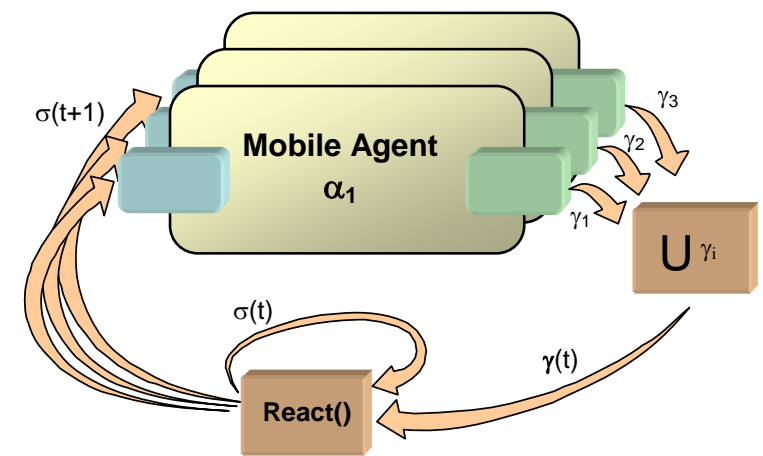

Fig. 4. Graphical representation of a MAS made up of hysteretic agents.

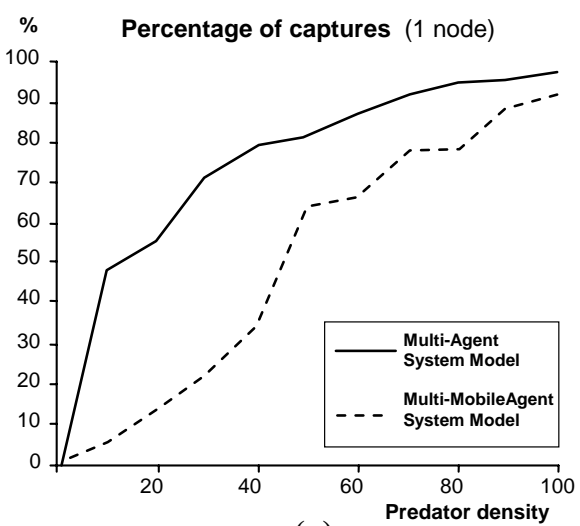

(a)

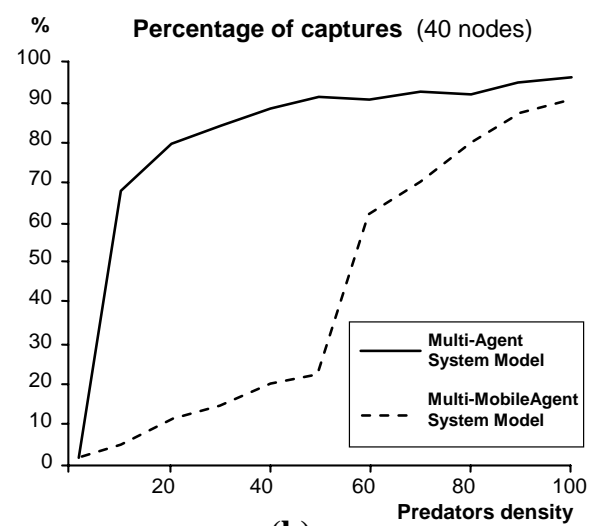

(b)

Fig. 5. Comparative of the capture percentages between the conventional model and the proposal of mobile agents applied to the predator-prey problem. (a) The game is developed in only one node. (b) The game is developed among forty nodes. 


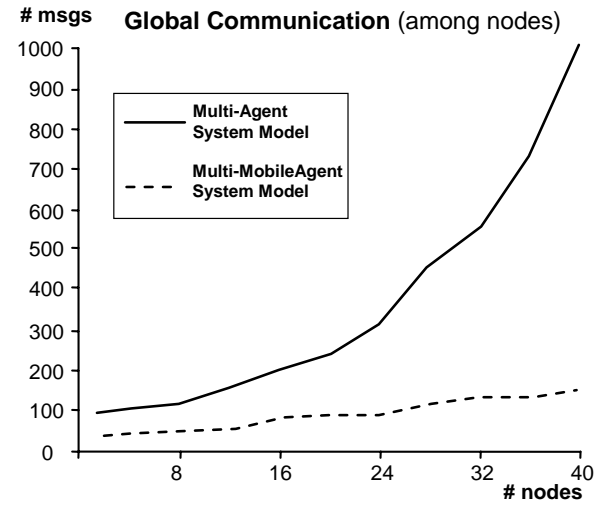

(a)

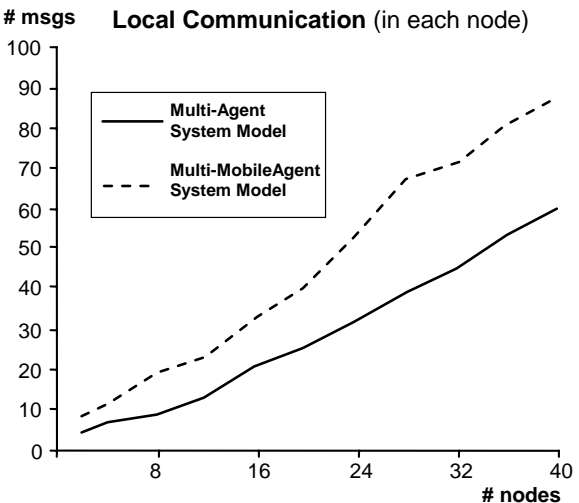

(b)

Fig. 6. (a) Number of messages originated by the predators among the different ecosystems. (b) Number of messages interchanged by the predators in an ecosystem. 